\title{
The Ivy Sign on Fluid Attenuated Inversion Recovery Images Related to Single-Photon Emission Computed Tomography Cerebral Blood Flow in Moyamoya Disease: A Case Report
}

\author{
Fumihiro MATANO ${ }^{1}$, Yasuo MURAI ${ }^{2}$, Asami KUBOTA ${ }^{1}$, Takayuki MIZUNARI ${ }^{1}$, Shiro KOBAYASHI ${ }^{1}$, Akio MORITA² \\ ${ }^{1}$ Chiba Hokusou Hospital, Department of Neurosurgery, Chiba, Japan \\ ${ }^{2}$ Nippon Medical School, Department of Neurological Surgery, Tokyo, Japan
}

Corresponding author: Fumihiro MATANO s00-078@nms.ac.jp

\section{ABSTRACT}

\begin{abstract}
Moyamoya disease is an idiopathic progressive cerebrovascular steno-occlusive disorder characterized by the formation of numerous collaterals called Moyamoya vessels. Accurate evaluation of vascular status and cerebral blood flow (CBF) is needed for prompt treatment to prevent ischemic and/or hemorrhagic events. The pathogenesis of the ivy sign on fluid attenuated inversion recovery (FLAIR) images of Moyamoya disease patients is unclear. We report a Moyamoya disease case wherein the ivy sign changed in relation to single-photon emission computed tomography (SPECT)-measured CBF during progression and following treatment. A 49-year-old female presented with slight aphasia and right hemiparesis. Magnetic resonance imaging (MRI) diffusionweighted image revealed cerebral infarction in the left frontal lobe. Cerebral angiography images showed bilateral distal internal carotid artery stenosis and Moyamoya vessels. FLAIR images exhibited the ivy sign. We performed superficial temporal arterymiddle cerebral artery (STA-MCA) bypass surgery with encephalogaleosynangiosis (EGS) and encephalomyosynangiosis (EMS) on the left side 6 months after first presentation. After operation, left-side resting CBF gradually improved on SPECT and the ivy sign decreased. On the other hand, right-side CBF gradually deteriorated at rest, and the ivy sign increased. Therefore, we performed STA-MCA bypass with EGS and EMS on the right side 4 years after first presentation. After the operation, the resting CBF increased and the ivy sign decreased. The FLAIR ivy sign may be a useful indicator of both deterioration and improvement of CBF status without the need for CBF imaging using contrast material.
\end{abstract}

KEYWORDS: Moyamoya disease, FLAIR, Ivy sign, SPECT

ABBREVIATIONS: CBF: Cerebral blood flow, EGS: Encephalogaleosynangiosis, EMS: Encephalomyosynangiosis, FLAIR: Fluid attenuated inversion recovery, ICA: Internal carotid artery, MCA: Middle cerebral artery, MRA: Magnetic resonance angiography, MRI: Magnetic resonance imaging, SPECT: Single-photon emission computed tomography, STA: Superficial temporal artery, PET: Positron emission tomography

\section{INTRODUCTION}

$\mathrm{M}$ oyamoya disease is an idiopathic progressive cerebrovascular steno-occlusive disorder characterized by the formation of numerous collaterals called "Moyamoya vessels" (17). Cerebral angiography and mag- netic resonance imaging (MRI) are indicated for the diagnosis and grading of Moyamoya disease (6), and single-photon emission computed tomography (SPECT) has also been used to evaluate cerebral blood flow (CBF)(3). The ivy sign, observed on post-contrast magnetic resonance images (MRI) and fluid attenuated inversion recovery (FLAIR) images, is
Fumihiro MATANO

Yasuo MURAI

Asami KUBOTA
(D) : $0000-0002-0760-5024$
(D) : $0000-0002-9753-1873$
(D) : $0000-0002-9435-1869$
Takayuki MIZUNARI Shiro KOBAYASHI Akio MORITA
(D) : $0000-0003-3216-4432$
(I) : $0000-0002-7500-7894$
(D) : $0000-0002-2497-5772$ 
speculated that the slow retrograde flow of engorged pial collateral arteries, the thickening of leptomeninges because of congestion, and maximally dilated pial vessels (14). Several studies have reported an improved ivy sign on MRI after bypass surgery $(12,13)$. The authors reported increased ivy sign with decreased quantitative CBF on $\mathrm{N}$-isopropyl- p-[(123)I] iodoamphetamine (123)I-IMP SPECT and decreased ivy sign with increased CBF following STA-MCA anastomosis over the course of adult Moyamoya disease.

However, the details of changes in the ivy signs are unclear. Here, we report a Moyamoya case with repeated reciprocal ivy sign changes relative to SPECT-measured CBF.

\section{CASE REPORT}

A 49-year-old female presented with slight aphasia and right hemiparesis. Blood pressure was $134 / 72 \mathrm{~mm} \mathrm{Hg}$ and pulse was 66 beats/minute with normal rhythm. The patient had a history of hypertension, but she neither smoked nor drank alcohol. Her mother had a history of cerebral infarction due to Moyamoya disease.
MRI diffusion-weighted image (DWI) revealed cerebral infarction in the left frontal lobe (Figure 1A). Magnetic resonance angiography (MRA) and cerebral angiography images showed bilateral distal internal carotid artery (ICA) stenosis and Moyamoya vessels on both sides of the ICA (Figure 1C, D). FLAIR images exhibited the ivy sign (Figure 1B).

The patient was administered conservative therapy for cerebral infarction and underwent rehabilitation for aphasia and hemiparesis. After rehabilitation, her deficits were improved. We performed superficial temporal artery (STA)-middle cerebral artery (MCA) bypass surgery with encephalogaleosynangiosis (EGS) and encephalomyosynangiosis (EMS) on the left side 6 months after first presentation.

After the left-side operation, CBF gradually improved at rest SPECT CBF and the ivy sign decreased (Figure 2A, B). On the other hand, right-side CBF gradually deteriorated at rest, and SPECT CBF and the ivy sign increased. Therefore, we performed STA-MCA bypass with EGS and EMS on the right side 4 years after first presentation. After operation, she had no neurological deficits, and SPECT CBF increased at rest and
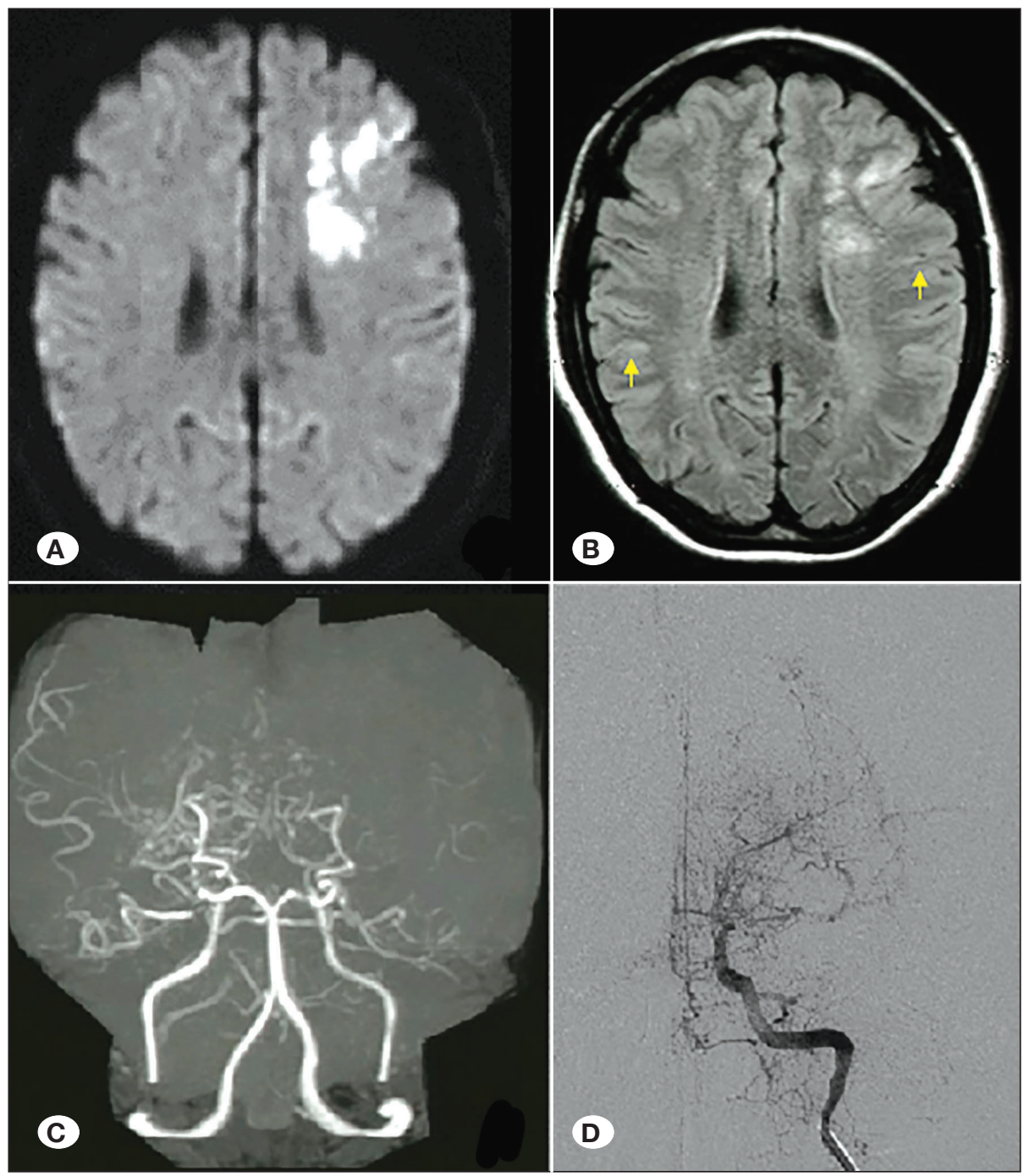

Figure 1: A) MRI-DWI revealed fresh cerebral infarction in left frontal lobe.

B) MRI FLAIR images showed the ivy sign.

C) MRA revealed bilateral ICA stenosis.

D) Typical net-like Moyamoya vessels observed by angiography. 
ivy sign decreased (Figure 3). No cerebral ischemic episodes were observed during the following year after two times of operation.

\section{DISCUSSION}

In the present study, the FLAIR ivy sign is a useful indicator for the observation of the state of CBF in Moyamoya disease because it is related to SPECT CBF.

Moyamoya disease, first reported in Japan in 1963 (17), is a rare cause of ischemic and hemorrhagic stroke with approximately $10 \%$ of cases due to inheritance (7). It is a progressive cerebrovascular disorder of the distal ICA accompanied by formation of net-like collateral vessels at the base of the brain. Disease progression can be divided into six stages $(18,19)$. There are currently no effective drug therapies for Moyamoya disease, and therefore, surgical revascularization by STAMCA anastomosis with or without an indirect bypass is the standard surgical treatment. As the increase in CBF achieved by indirect revascularization is often unsatisfactory, and direct revascularization is usually feasible, particularly in adults
(1), direct revascularization is recommended by Japanese guidelines for the treatment of Moyamoya disease (6). However, accurate evaluation of vascular status and CBF is needed for prompt treatment to prevent ischemic and/or hemorrhagic events. For evaluating the stages of Moyamoya disease, cerebral angiography is still the gold standard; however, with the continued development of MRI techniques, diagnosis of Moyamoya disease with a $>1.5$ Tesla magnet has become possible $(4,5,9,21)$. SPECT and positron emission tomography (PET) have also been used to evaluate $\operatorname{CBF}(3,15)$. Moreover, leptomeningeal high-signal intensity on post-contrast T1weighted MR imaging, the ivy sign is assumed to indicate decreased cerebral perfusion, dilated pial vasculature, and slow flow in developed leptomeningeal collaterals. The ivy sign is also observed on unenhanced FLAIR images (14). In addition, the ivy sign correlated with neurological deficit and previous brain old lesions, and reflected the progression of Moyamoya disease $(2,20)$.

Kaku et al. reported a positive correlation between ivy sign proliferation and cerebral blood volume (CBV) in Moyamoya disease, and a more obvious negative correlation was found

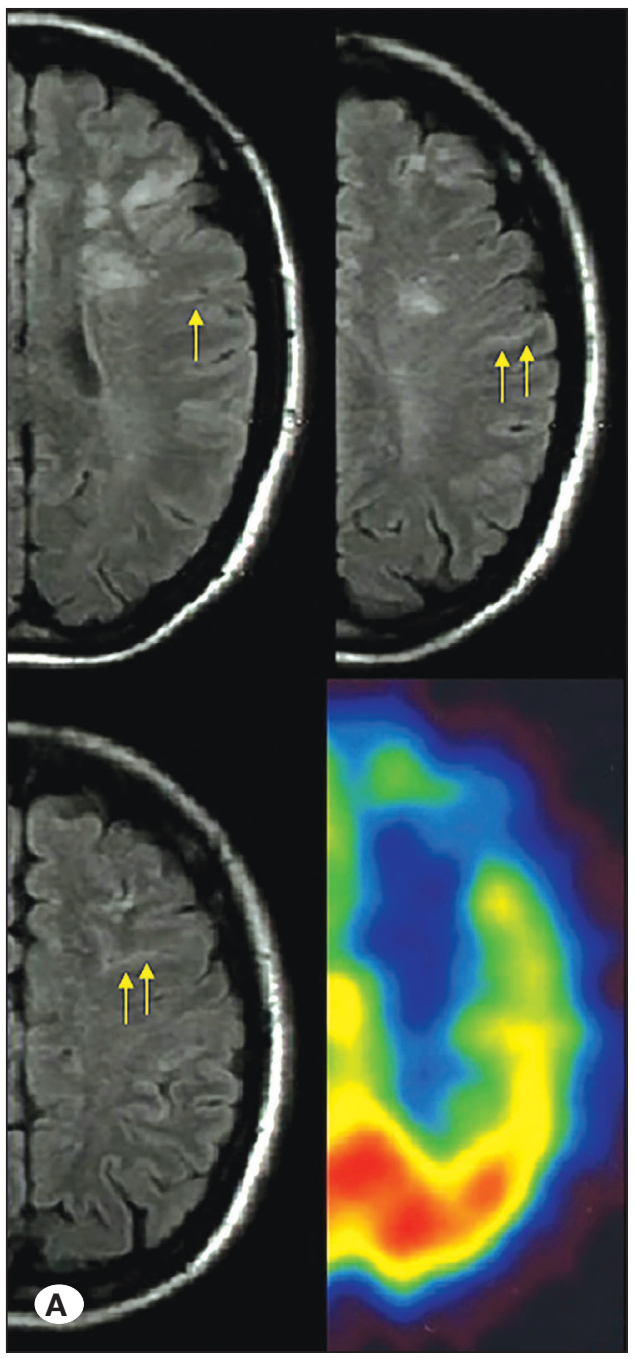

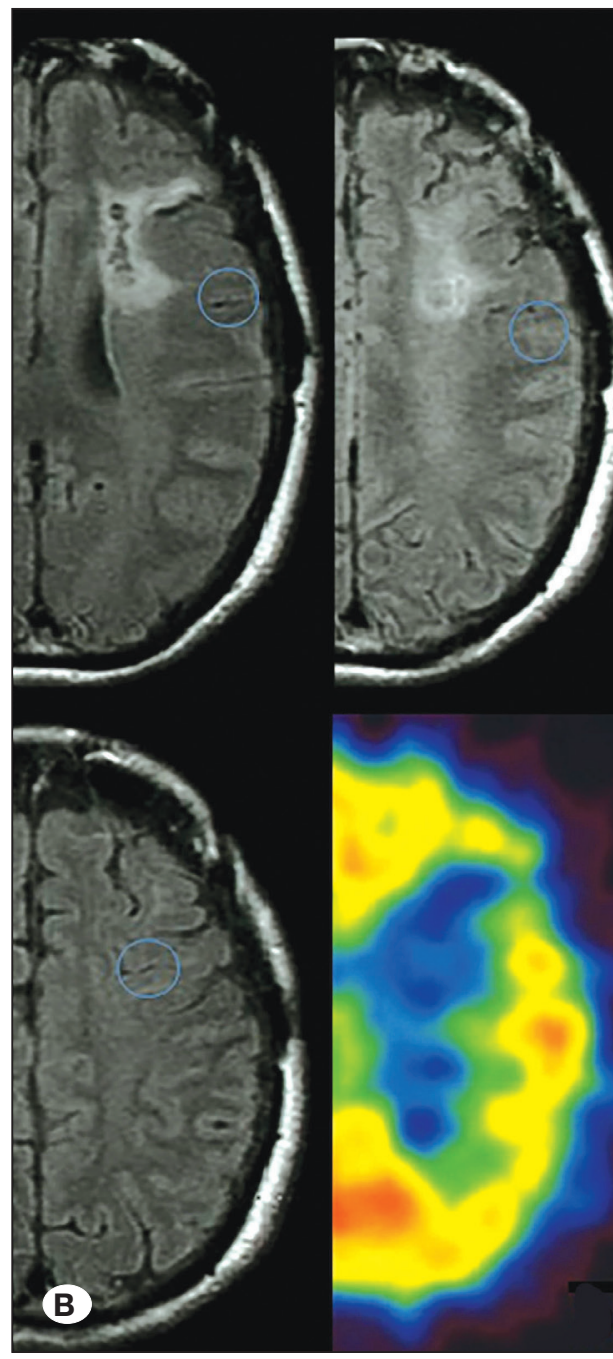

Figure 2:

A) Pre-operative FLAIR images showing the ivy sign and SPECT showing hypoperfusion in the left frontal lobe.

B) Post-operative FLAIR images showing decreased ivy sign in left frontal lobe, and SPECT revealed increased CBF in left frontal lobe. 


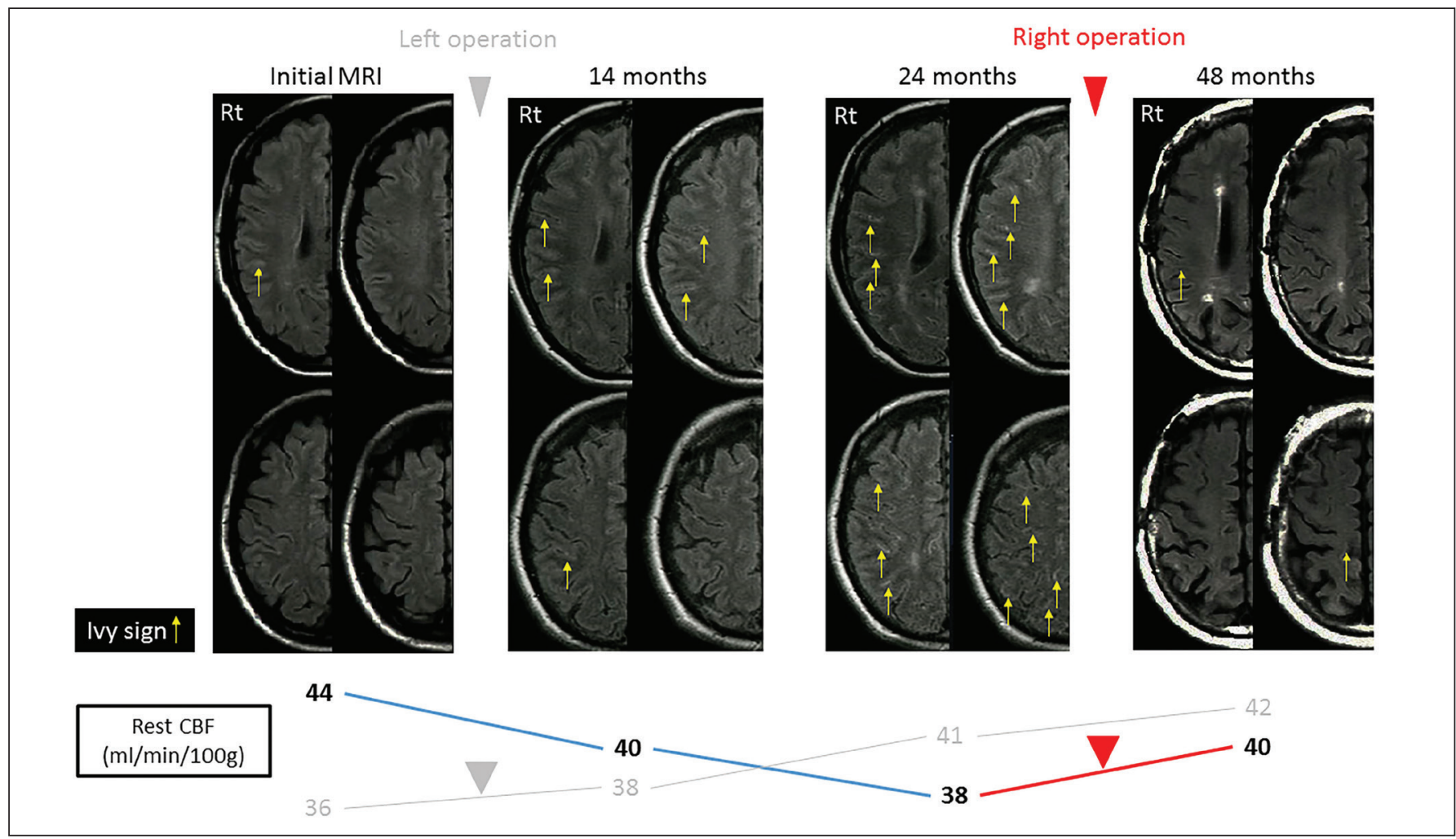

Figure 3: Follow-up ivy sign and relation to SPECT CBF.

between ivy sign proliferation and CBF/CBV (11). A past report found that disappearance of the ivy sign indicated hemodynamic improvement after revascularization surgery (10). Further, Lee et al. reported that the ivy sign on FLAIR is a useful indicator of brain hemodynamic changes following surgery in adult Moyamoya disease (13). However, there are no reports of a FLAIR ivy sign increase during follow-up related to a decrease in quantitative CBF.

In Moyamoya disease, the main aim of surgery is to prevent the brain from ischemic and hemorrhagic events. Thus, careful and regular postoperative hemodynamic evaluation is necessary. Therefore, simple evaluation modalities without contrast material, such as SPECT or PET, could be advantageous. In the present case, the FLAIR ivy sign was inversely related to CBF as measured by SPECT. We suggest that the FLAIR ivy sign is useful for follow-up imaging and helpful for decisions related to surgical timing of Moyamoya disease because it obviates risks associated with catheterization and contrast agents.

In addition, when Moyamoya disease patients are admitted to emergency department with headache or transient disturbance of consciousness, FLAIR images may be a useful indicator to evaluate the hemodynamic condition such as cerebrovascular reactivity and degree of ivy sign can suggest the need for more aggressive medical treatment or revascularization surgery (16).
Horie et al. reported that de novo ivy sign could indicate cerebral hyperperfusion after revascularization procedure, and the ivy sign is therefore a useful tool for risk management postoperatively (8).

Additional cases are required to confirm this relationship between the FLAIR ivy sign and SPECT-measured CBF.

\section{CONCLUSION}

Ivy sign on FLAIR images is related to SPECT CBF. Therefore, the FLAIR ivy sign is a useful indicator for follow-up imaging in Moyamoya disease.

\section{REFERENCES}

1. Bao XY, Duan L, Yang WZ, Li DS, Sun WJ, Zhang ZS, Zong $\mathrm{R}$, Han C: Clinical features, surgical treatment, and long-term outcome in pediatric patients with Moyamoya disease in China. Cerebrovasc Dis 39:75-81, 2015

2. El Beltagi AH, El-Sheikh A, El-Saif R, Norbash A: Ivy sign in mildly symptomatic beta-thalassemia intermedia, with development of moyamoya disease. Neuroradiol J 27:23-28, 2014

3. Feole JB, Ali A, Fordham EW, Huckman M, Shenker DM: Serial SPECT imaging in moyamoya using I-123 IMP. A method of noninvasive evaluation and follow-up. Clin Nucl Med 18:4345, 1993 
4. Fukui M: Guidelines for the diagnosis and treatment of spontaneous occlusion of the circle of Willis ('moyamoya' disease). Research Committee on Spontaneous Occlusion of the Circle of Willis (Moyamoya Disease) of the Ministry of Health and Welfare, Japan. Clin Neurol Neurosurg 99 Suppl 2:S238-240, 1997

5. Fushimi Y, Miki Y, Kikuta K, Okada T, Kanagaki M, Yamamoto A, Nozaki K, Hashimoto N, Hanakawa T, Fukuyama H, Togashi $\mathrm{K}$ : Comparison of 3.0- and 1.5-T three-dimensional time-offlight MR angiography in Moyamoya disease: Preliminary experience. Radiology 239:232-237, 2006

6. Hashimoto N, Tominaga T, Miyamoto S, Nagata I, Houkin K, Suzuki N, Koizumi A, Nogawa S, Nakagawara J, Kitagawa K, Kuroda S, Kikuta K, Fujimura M, Takahashi J, Hayashi K, Oki $\mathrm{K}$, Hoshino H, Takagi Y; Research Committee on the Pathology and Treatment of Spontaneous Occlusion of the Circle of Willis; Health Labour Sciences Research Grant for Research on Measures for Infractable Diseases: Guidelines for diagnosis and treatment of moyamoya disease (spontaneous occlusion of the circle of Willis). Neurol Med Chir (Tokyo) 52:245-266, 2012

7. Hayashi K, Horie N, Izumo T, Nagata I: A nationwide survey on unilateral moyamoya disease in Japan. Clin Neurol Neurosurg 124:1-5, 2014

8. Horie N, Morikawa M, Morofuji Y, Hiu T, Izumo T, Hayashi K, Nagata I: De novo ivy sign indicates postoperative hyperperfusion in moyamoya disease. Stroke 45:1488-1491, 2014

9. Houkin K, Aoki T, Takahashi A, Abe H: Diagnosis of moyamoya disease with magnetic resonance angiography. Stroke 25:2159-2164, 1994

10. Ideguchi R, Morikawa M, Enokizono M, Ogawa Y, Nagata I, Uetani M: Ivy signs on FLAIR images before and after STAMCA anastomosis in patients with Moyamoya disease. Acta Radiol 52:291-296, 2011

11. Kaku Y, lihara K, Nakajima N, Kataoka H, Fukushima K, lida H, Hashimoto N: The leptomeningeal ivy sign on fluid-attenuated inversion recovery images in moyamoya disease: Positron emission tomography study. Cerebrovasc Dis 36:19-25, 2013
12. Kawashima M, Noguchi T, Takase Y, Nakahara Y, Matsushima T: Decrease in leptomeningeal ivy sign on fluid-attenuated inversion recovery images after cerebral revascularization in patients with Moyamoya disease. AJNR Am J Neuroradiol 31:1713-1718, 2010

13. Lee JK, Yoon BH, Chung SY, Park MS, Kim SM, Lee do S: The usefulness of the ivy sign on fluid-attenuated intensity recovery images in improved brain hemodynamic changes after superficial temporal artery-middle cerebral artery anastomosis in adult patients with moyamoya disease. $J$ Korean Neurosurg Soc 54:302-308, 2013

14. Maeda M, Tsuchida C: "Ivy sign" on fluid-attenuated inversionrecovery images in childhood moyamoya disease. AJNR Am J Neuroradiol 20:1836-1838, 1999

15. Seo KD, Suh SH, Kim YB, Kim JH, Ahn SJ, Kim DS, Lee KY: Ivy sign on fluid-attenuated inversion recovery images in Moyamoya disease: Correlation with clinical severity and old brain lesions. Yonsei Med J 56:1322-1327, 2015

16. Sivrioglu AK, Saglam M, Yildiz B, Anagnostakou V, Kizilkilic $\mathrm{O}:$ Ivy sign in Moyamoya disease. Eurasian J Med 48:58-61, 2016

17. Suzuki J, Takaku A: Cerebrovascular "moyamoya" disease. Disease showing abnormal net-like vessels in base of brain. Arch Neurol 20:288-299, 1969

18. Takanashi J: Moyamoya disease in children. Brain Dev 33:229234, 2011

19. Takeuchi K: Moyamoya phenomenon and Moyamoya diseases (author's transl). No To Shinkei 30:1183-1191, 1978 (In Japanese)

20. Vuignier S, Ito M, Kurisu K, Kazumata K, Nakayama N, Shichinohe H, Shiga T, Kiss JZ, Tamaki N, Houkin K: Ivy sign, misery perfusion, and asymptomatic moyamoya disease: FLAIR imaging and (15)O-gas positron emission tomography. Acta Neurochir (Wien) 155:2097-2104, 2013

21. Yamada I, Suzuki S, Matsushima Y: Moyamoya disease: Comparison of assessment with MR angiography and MR imaging versus conventional angiography. Radiology 196:211218, 1995 